\title{
A Microdosimetric Analysis of Absorbed Dose to Tumor as a Function of Number of Microspheres per Unit Volume in ${ }^{90} \mathrm{Y}$ Radioembolization
}

\author{
Alexander S. Pasciak ${ }^{1,2}$, Austin C. Bourgeois ${ }^{1,3}$, and Yong C. Bradley ${ }^{1}$ \\ ${ }^{1}$ Department of Radiology, University of Tennessee Graduate School of Medicine, Knoxville, Tennessee; ${ }^{2}$ School of Medicine, Johns \\ Hopkins Hospital, Baltimore, Maryland; and ${ }^{3}$ Department of Radiology, Medical University of South Carolina, Charleston, South \\ Carolina
}

\begin{abstract}
Differences in maximum tolerable absorbed dose to normal liver between ${ }^{90} \mathrm{Y}$ radioembolization and external-beam radiation therapy have been explained by citing differences in absorbed-dose heterogeneity at the microscopic level. We investigated microscopic absorbed-dose heterogeneity in radioembolization as a function of the number of microspheres per unit volume in tumor. The goal was to determine what effect the number of microspheres may have, if any, on tumor control in ${ }^{90} \mathrm{Y}$ radioembolization. Methods: ${ }^{90} \mathrm{Y}$ PET/CT data were combined with microscopic probability-density functions describing microsphere clustering to provide realistic simulation using Monte Carlo modeling on both a macroscopic and a microscopic level. A complete microdosimetric analysis using $100-\mu \mathrm{m}$ voxels was performed on the basis of ${ }^{90} \mathrm{Y} \mathrm{PET/CT}$ data from 19 tumors treated using radioembolization. Simulations were performed with average tumor microsphere-number densities from 200 to 70,000 spheres $/ \mathrm{mL}$. Monte Carlo simulations of each tumor and number density were repeated 20 times to establish SE. A 2-way balanced ANOVA was used to determine whether differences in microsphere-number density affected common tumor-dose metrics. Results: Decreasing the microsphere-number density resulted in a decrease in $D_{70}$, the minimum dose to $70 \%$ of the tumor. The slope of the dose-volume histogram also decreased with decreasing microsphere-number density in all tumors. Compared with a density of 50,000 spheres $/ \mathrm{mL}$, decreases in $D_{70}$ were statistically significant below 20,000 spheres $/ \mathrm{mL}$. However, these differences are unlikely to have clinical significance until the density decreases to below 5,000 spheres $/ \mathrm{mL}$. Although $D_{70}$ was decreased at a low microsphere-number density, one can compensate for decreases by an increase in the average tumorabsorbed dose, that is, by increasing the radioembolization treatment dose. Conclusion: Differences in microsphere-number density may have an effect on microscopic tumor absorbed-dose inhomogeneity. These results begin to explain differences in treatment planning strategies between glass and resin radioembolization devices.
\end{abstract}

Key Words: yttrium-90; 90 Y; radioembolization; interventional oncology

J Nucl Med 2016; 57:1020-1026

DOI: 10.2967/jnumed.115.163444

\footnotetext{
Received Jul. 6, 2015; revision accepted Feb. 1, 2016.

For correspondence or reprints contact: Alexander S. Pasciak, Johns Hopkins School of Medicine, 733 N. Broadway, Baltimore, MD 21205.

E-mail: alexander.pasciak@gmail.com

Published online Feb. 18, 2016.

COPYRIGHT (c) 2016 by the Society of Nuclear Medicine and Molecular Imaging, Inc.
}

$\mathbf{O}$ ver the past 20 years, the worldwide use of ${ }^{90} \mathrm{Y}$ radioembolization for treatment of primary and metastatic liver cancer has grown significantly. Glass (TheraSphere; BTG International) and resin (SIR-Spheres; SIRTeX Medical) microspheres are the most widely used-and therefore the most frequently comparedradioembolization devices. In particular, differences in the maximum tolerable absorbed dose to normal liver between these devices has been the source of an interesting paradox. Recommendations on the maximum normal-liver dose for radioembolization differ significantly between glass and resin devices, with $70 \mathrm{~Gy}$ suggested as a maximum for resin microspheres (1) and $120 \mathrm{~Gy}$ for glass microspheres (2). This paradox is confounded further when one also considers the 30-Gy toxicity threshold for wholeliver external-beam irradiation (3).

The fundamental explanation for the difference between the maximum tolerable normal-liver dose from external-beam radiation therapy and radioembolization is the heterogeneity of absorbed dose at a microscopic level. Although external-beam radiation therapy will produce uniform absorbed doses at a microscopic level, this is not the case with radioembolization. Fox et al. first suggested that the microscopic absorbed-dose inhomogeneity of radioembolization may spare microscopic areas of normal liver between microspheres, leading to differences in toxicity between radioembolization and external-beam radiation therapy (3). The dose-inhomogeneity argument has also been applied to explain the paradox of differences in the maximum tolerable normal-liver dose between glass and resin microspheres based on their physical and radiologic characteristics (Table 1).

Resin and glass microspheres have calibrated specific activities of 50 and 2,500 Bq/sphere, respectively (4), leading to a potential difference in the number of microspheres infused to achieve a specific dosimetric endpoint (Table 1). Recently, Walrand et al. (5) explored normal-liver absorbed-dose inhomogeneity using a 3-dimensional (3D) model of a section of normal liver tissue based on a repeating arrangement of liver lobules. Although several previous authors $(6-8)$ had performed similar computational analyses of liver lobule vasculature and microsphere deposition, the study of Walrand et al. was unique (5) in that they used unequal trapping to show that glass microspheres spare critical tissues in normal liver, that is, the portal triad. This work offered a potential explanation for differences in maximum tolerable normal-liver dose between resin and glass microspheres. Further, ${ }^{90} \mathrm{Y}$ PET/CT and its superior resolution allowed verification of the microscopic simulation of Walrand 
TABLE 1

Overview of Resin and Glass ${ }^{90}$ Y Radioembolization Products

\begin{tabular}{|c|c|c|}
\hline Characteristic & SIR-Spheres & TheraSphere \\
\hline Composition & Resin with ${ }^{90} \mathrm{Y}$ bound to surface & Glass permanently impregnated with ${ }^{90} \mathrm{Y}$ \\
\hline Size & $32.5 \pm 5 \mu \mathrm{m}$ & $20-30 \mu \mathrm{m}$ \\
\hline Number of spheres per vial & 40-80 million & 1.2-8 million \\
\hline Specific activity at calibration & $50 \mathrm{~Bq} / \mathrm{sphere}$ & $2500 \mathrm{~Bq} / \mathrm{sphere}$ \\
\hline $\begin{array}{l}\text { Time after infusion when } 90 \% \text { of absorbed } \\
\text { dose is delivered }\end{array}$ & $\sim 9 \mathrm{~d}$ & $\sim 9 \mathrm{~d}$ \\
\hline Shelf life from time of calibration & $24 \mathrm{~h}$ & $12 \mathrm{~d}$ \\
\hline Specific activity at treatment & $50-38.6^{\star} \mathrm{Bq} / \mathrm{sphere}$ & $2,500-111^{*} \mathrm{~Bq} / \mathrm{sphere}$ \\
\hline $\begin{array}{l}\text { Range of microsphere-number density per } \\
\text { gray of average tumor-absorbed dose }\end{array}$ & $413-535 \mathrm{~mL}-\mathrm{Gy}^{-1}$ & 8.2-186 mL-Gy ${ }^{-1}$ \\
\hline \multicolumn{3}{|l|}{ Range of microsphere-number density at... } \\
\hline 100-Gy average tumor-absorbed dose & $41,300-53,500 \mathrm{~mL}^{-1}$ & $820-18,600 \mathrm{~mL}^{-1}$ \\
\hline 250-Gy average tumor-absorbed dose & $103,250-133,750 \mathrm{~mL}^{-1}$ & $2,050-46,500 \mathrm{~mL}^{-1}$ \\
\hline \multicolumn{3}{|c|}{$\begin{array}{l}{ }^{*} \text { Based on decay at expiration/shelf-life of product. } \\
{ }^{\dagger} \text { Based on clinical specific activity range. } \\
\text { Adapted from Salem and Thurston (4). }\end{array}$} \\
\hline
\end{tabular}

et al. by confirming macroscopic deposition variations in real and simulated radioembolization procedures (5).

Previous microdosimetric simulations of radioembolization (5-8) have considered only an ideal model of normal liver, with no analysis of tumor tissue. This restriction was primarily due to the limited information known about tumor vascular microanatomy, which has been observed only histologically $(8,9)$. However, as shown by Walrand et al. (5), the resolution of ${ }^{90} \mathrm{Y}$ PET/CT may be high enough to bridge microscopic and macroscopic microsphere distribution in vivo. In the current work, ${ }^{90} \mathrm{Y}$ PET/CT data have been combined with probability-density functions (PDFs) describing microscopic microsphere tumor deposition to provide insight into the effect of microsphere-number density on both microscopic and macroscopic tumor dosimetry. These findings may explain why the treatment plan needs to differ between glass and resin radioembolization devices that have differences in specific activity per microsphere.

\section{MATERIALS AND METHODS}

Quantitative data describing the deposition patterns of radioembolic microspheres in tumor tissue are limited, although several authors have attempted histologic analysis (9-11). The detail with which these authors quantified their analyses varied, but two findings of the studies were concordant and have been incorporated into the current work. The first is that microspheres are not deposited randomly in the tumor; they are deposited in clusters (9-11). The second is that increasing the number of microspheres increases not the number of clusters but the number of spheres in each cluster (11).

In this work, Monte Carlo sampling techniques have been used to model the distribution of microspheres on both a microscopic and a macroscopic level. On a microscopic level, microsphere distribution was sampled from experimental data provided by Campbell et al. (9), derived from histologic analyses of biopsy specimen. Differential data describing the spectrum of distances between microsphere clusters, cluster diameters, and numbers of microspheres per cluster (9) were converted into PDFs and used in combination with ${ }^{90} \mathrm{Y}$ PET/CT data to determine microscopic microsphere distribution as a function of microsphere-number density. The first step in producing a simulation that provides concordance on both a macroscopic level and a microscopic level is to unify these data with a single data structure. To facilitate this, microsphere placement was performed within a $3 \mathrm{D}$ matrix of $100-\mu \mathrm{m}$ voxels, referred to as $\mu$-voxels to distinguish them from the larger PET/CT voxels (i.e., voxels) in the remainder of the article.

\section{Placing Spheres into PET/CT Voxels}

All ${ }^{90} \mathrm{Y}$ PET/CT data used in this work were acquired on a Biograph mCT Flow scanner (Siemens Healthcare) with resolution recovery and time-of-flight, ordered-subset expectation maximization (1 iteration and 21 subsets, an all-pass reconstruction filter, a $400 \times 400[2 \mathrm{~mm}]$ matrix, and continuous bed motion at $0.2 \mathrm{~mm} / \mathrm{s})$. With a size of $2 \mathrm{~mm}^{3}$, each voxel consisted of a matrix of $20 \times 20 \times 20 \mu$-voxels $(8,000 \mu$-voxels $)$. Data from ${ }^{90} \mathrm{Y}$ PET/CT were normalized into a 3D PDF used for macroscopic sampling of microsphere deposition, with the first step being the localization of a voxel for placement of a single microsphere. The rejection technique (12) was used to select a voxel based on the ${ }^{90} \mathrm{Y}$ PET/ CT 3D PDF as shown in the pseudocode in Equation 1.

$$
\begin{aligned}
\operatorname{while}\left(\mu_{1}\right. & >\operatorname{PET}(x, y, z)) \\
x & =400 \mu_{2} \\
y & =400 \mu_{3} \\
z & =400 \mu_{4},
\end{aligned}
$$

where $\mu_{x}$ is a uniformly distributed random number in the range [0,1], PET is a 3D normalized PDF obtained directly from ${ }^{90} \mathrm{Y}$ PET/CT, and $x, y, z$ are integers indexing the PET PDF. On acceptance of the routine in Equation 1, a microsphere then must be placed within that voxel subject to the microscopic deposition algorithm detailed in the next section.

\section{Placing Spheres into $\mu$-Voxels}

Within each voxel, a $\mu$-voxel was selected from a predetermined 3D microscopic probability matrix that included data for potential 
microsphere cluster locations and sizes and the relative probability that a microsphere will land within a cluster. The probability matrix was determined from PDFs based on biopsy specimens (9) using a randomly-growing-tree model to place cluster centers within the $3 \mathrm{D}$ volume subtended by all $\mu$-voxels. Successive cluster locations were determined using the rejection technique to sample the minimum distance between the new and previously placed clusters. A branching direction was then selected at random in $4 \pi$ steradians at a radius equal to the sampled distance to define the new cluster location relative to the previously placed cluster. The new cluster location was compared with all previously placed cluster locations to ensure that the sampled distance between clusters for the current cluster, and previous clusters, was not violated. If a violation occurred, a new previous cluster was selected at random and the process was repeated until cluster placement was successful. After each successful cluster placement, the cluster center location, cluster size, and relative probability that a microsphere will land within a cluster were stored in the probability matrix. A diagrammatic overview of these processes is provided in Figure 1.

Filling of predetermined clusters with individual microspheres was ultimately constrained macroscopically within each voxel by PET/CT data, and in each voxel the sphere was placed in a predetermined cluster by random sampling of the probability that spheres will land within that cluster, previously determined and stored in the probability matrix. At a microscopic level, extensive use of probability-sampled Monte Carlo techniques for sphere placement resulted in realistic distributions, even exhibiting variations similar to postradioemboliza-

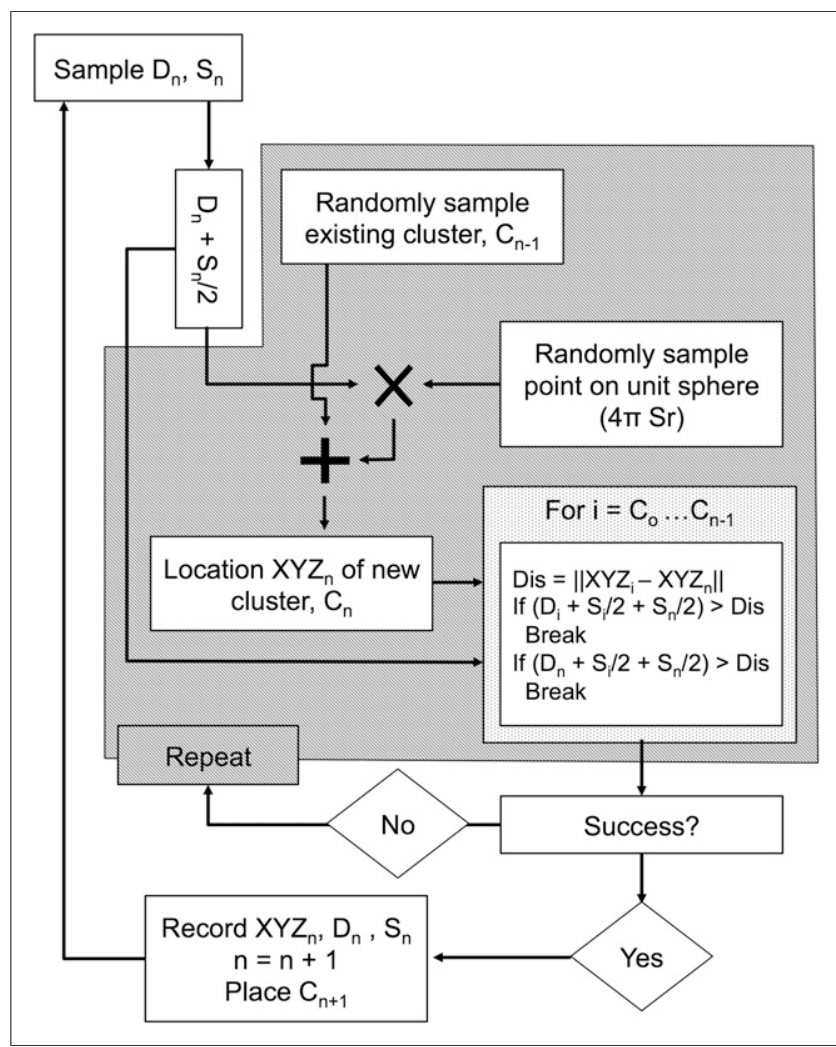

FIGURE 1. Simplified flowchart describing algorithm used to identify microsphere cluster locations and size. $\mathrm{C}=$ microsphere cluster; $\mathrm{D}=$ sampled distance between microsphere clusters; $\mathrm{S}=$ cluster size; $\|\mathrm{x}\|=$ vector norm operator; $\mathrm{XYZ}$ = vector describing spatial coordinates of cluster center. tion histologic specimens. Figure 2 shows a simulated slice of microscopic deposition with 50,000 spheres/mL.

In all simulations of microsphere deposition, the distribution pattern was not varied on the basis of microsphere composition or size (i.e., glass vs. resin). This decision was consistent with findings from specimens suggesting that microscopic deposition along an arterial tract is not notably different between glass and resin devices (10). Simulations were performed at average tumor microsphere-number densities ranging from 200 to 70,000 spheres $/ \mathrm{mL}$. Simulation increments of 200 and 1,000 spheres/mL were used from 200 to 5,000 and from 5,000 to 70,000 spheres $/ \mathrm{mL}$, respectively.

\section{Assessment of Impact of Microsphere-Number Density}

The impact of microsphere-number density was assessed using two approaches within the framework discussed above.

Uniform Approach. The first method was the uniform approach, which considers the case of a tumor with a homogeneous macroscopic distribution of activity. In this case, the probability that a microsphere would be deposited within a voxel was equal for all voxels. Microscopic distribution at the $\mu$-voxel level was performed as previously described based on sampling from the data of Campbell et al. (9).

Patient-Based Approach. The second method was the patientbased approach. The institutional review board of the primary site approved the patient data collection protocol for this prospective trial. Informed consent was obtained from all study participants, and each underwent postradioembolization ${ }^{90} \mathrm{Y}$ PET/CT. The enrollment criteria included patients with unresectable liver cancer clinically referred for hepatic ${ }^{90} \mathrm{Y}$ radioembolization with resin microspheres for both on-label and off-label treatment of their disease, with no sex or race restriction. Thirteen patients (aged $47-80 \mathrm{y})$ were enrolled for treatment of hepatocellular carcinoma $(n=8)$, liver-dominant metastatic disease $(n=4)$, or intrahepatic cholangiocarcinoma $(n=1)$. The median MELD score (model for

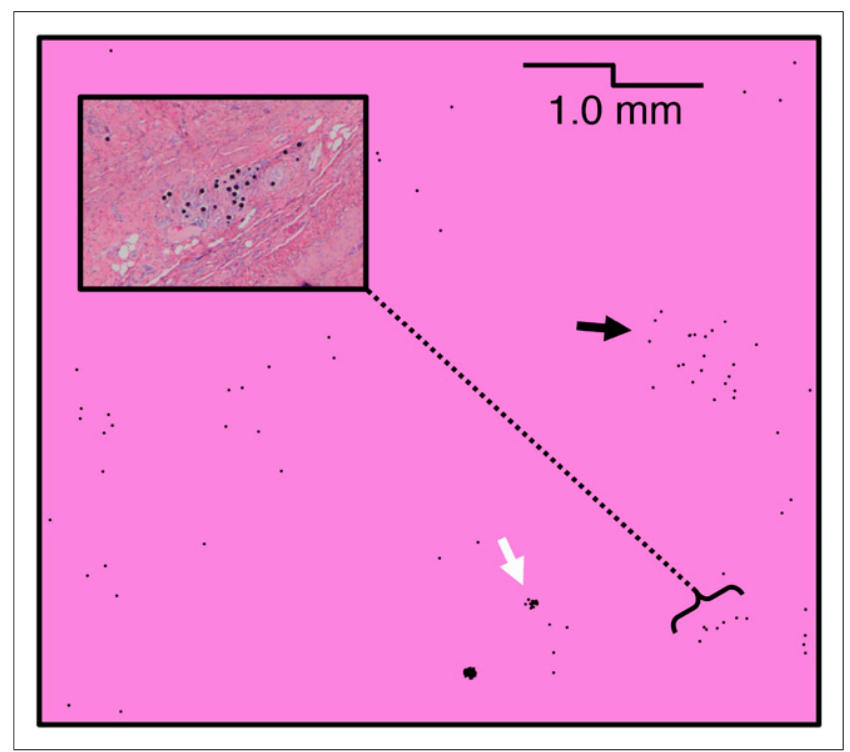

FIGURE 2. Microscopic deposition of spheres at number density of 50,000 spheres $/ \mathrm{mL}$. Small clusters (white arrow) and large clusters (black arrow) are apparent. Monte Carlo process allows for realistic microscopic distribution consistent with experimental findings, including oblique clusters. For comparison, expanded section shows microscopic view of oblique cluster of resin microspheres surrounding arteriole in animal tissue. 
end-stage liver disease) was 7 (range, 5-17), with 3 of 13 patients having cirrhosis classified as Child-Pugh score A, 3 as score B, and none as score $\mathrm{C}$. Segmental tumor-associated portal vein thrombus was present in 3 patients. In total, 19 individual lesions were considered (mean, 1.45 per patient), with a mean maximum tumor diameter of $5.6 \mathrm{~cm}$ (range, 1.6-15.0 cm). Additional demographic data are available in Table 2 .

Individual lesions $(n=19)$ were contoured in 3 dimensions under the supervision of a board-certified nuclear medicine radiologist. The voxel matrix included 5 padding voxels of normal liver tissue around all tumor edges to establish realistic boundary conditions and chargedparticle equilibrium.

\section{Radiation Transport and Dosimetry}

Although the Monte Carlo method was used to place spheres within $\mu$-voxels, ensuing radiation transport calculations were performed analytically using a predetermined radiation-dose point kernel, which, for 100- $\mu \mathrm{m}$ voxels, was computed using MCNP-X, version 2.5.0 (13), with the ${ }^{90} \mathrm{Y} \beta$-spectrum as defined by Eckerman et al. (14) in a $24 \times$ $24 \times 24 \mathrm{~mm}$ block of liver tissue (15). Electron-photon coupled transport simulation of primary and secondary particles was performed with an energy cutoff of $1 \mathrm{keV}$. Simulation of $5 \times 10^{8}$ histories was completed in $53 \mathrm{~h}$ on an E5 workstation (Intel) with $32 \mathrm{~GB}$ of randomaccess memory. Convolution of the radiation-dose point kernel with all $\mu$-voxel datasets was performed in the frequency domain using the fast-Fourier-transform algorithm. So that results could be applied to many clinical scenarios, all resulting dose distributions were normalized as a function of average absorbed dose either to the tumor as a whole or to the entire volume in the case of the uniform approach. The average absorbed dose $\left(D_{\text {avg }}\right)$, specific activity per microsphere $\left(A_{s}\right)$,

TABLE 2

Summary of ${ }^{90} \mathrm{Y}$ PET/CT Patient Data Used

\begin{tabular}{lrll}
\hline Patient & Tumor & \multicolumn{1}{c}{ Type } & Size $(\mathrm{cm})$ \\
\hline 1 & 1 & Hepatocellular carcinoma & $7 \times 9.2 \times 11$ \\
\hline 2 & 2 & Adenocarcinoma & $1.9 \times 2.5 \times 4.2$ \\
\hline 3 & 3 & Adenocarcinoma & $3.8 \times 3.4 \times 3.4$ \\
\hline 4 & 4 & Hepatocellular carcinoma & $4.4 \times 4.6 \times 3.1$ \\
\hline 5 & 5 & Cholangiocarcinoma & $3.1 \times 2.3 \times 2.8$ \\
\hline 5 & 6 & Cholangiocarcinoma & $4.7 \times 5.8 \times 6.1$ \\
\hline 5 & 7 & Cholangiocarcinoma & $3.0 \times 3.1 \times 2.3$ \\
\hline 6 & 8 & Breast cancer & $2.7 \times 2.3 \times 2.3$ \\
\hline 7 & 9 & Hepatocellular carcinoma & $15 \times 9.4 \times 10.4$ \\
\hline 8 & 10 & Hepatocellular carcinoma & $9.3 \times 7.1 \times 6.8$ \\
\hline 9 & 11 & Hepatocellular carcinoma & $2.1 \times 2.5 \times 3.1$ \\
\hline 9 & 12 & Hepatocellular carcinoma & $2.8 \times 2.9 \times 2.1$ \\
\hline 9 & 13 & Hepatocellular carcinoma & $3.1 \times 3.2 \times 3.5$ \\
\hline 9 & 14 & Hepatocellular carcinoma & $2.8 \times 3.1 \times 2.9$ \\
\hline 10 & 15 & Hepatocellular carcinoma & $8.7 \times 8.9 \times 12.3$ \\
\hline 11 & 16 & Neuroendocrine & $1.1 \times 1.3 \times 2.6$ \\
\hline 11 & 17 & Neuroendocrine & $2.6 \times 2.0 \times 1.2$ \\
\hline 12 & 18 & Hepatocellular carcinoma & $4.9 \times 6.6 \times 9.1$ \\
\hline 13 & 19 & Hepatocellular carcinoma & $6.2 \times 4.5 \times 5.1$ \\
\hline & & & \\
\hline
\end{tabular}

All tumors were treated with resin microspheres, and none of the tumors reached stasis. and average microsphere-number density $(N)$ are related as shown in Equation 2:

$$
\mathrm{D}_{\mathrm{avg}}(\mathrm{Gy})=\frac{\mathrm{A}_{\mathrm{s}}(\mathrm{Bq}) \cdot N\left(\mathrm{~mL}^{-1}\right) \cdot 4.986 \cdot 10^{-5}(\mathrm{~J} \cdot \mathrm{s})}{1.03(\mathrm{~g} / \mathrm{mL})} . \quad \text { Eq. } 2
$$

Using Equation 2, the average number density given a known average absorbed dose and specific activity can be determined. The available specific-activity range of the radioembolization device used (Table 1) will define the range of average absorbed dose that can be achieved at a given number density.

\section{Analysis and Statistics}

Several figures of merit were selected to reduce multidimensional dosimetric data into a presentable form. For each tumor, $\mathrm{D}_{70}, \mathrm{D}_{90}$, and $\mathrm{D}_{95}$ were acquired as a function of microsphere-number density, where $\mathrm{D}_{70}$ is the minimum dose to $70 \%$ of the tumor volume (16). A total of 20 complete runs for each tumor, including resampling of all cluster locations at every number density, was performed to establish SEs due to random variance from the Monte Carlo method. $\mathrm{D}_{70}$ data from 20 Monte Carlo runs of each tumor (Table 2) were analyzed using a 2-way balanced ANOVA. Data at each number density were compared with data computed at a number density of 50,000 spheres/ $\mathrm{mL}$ to examine whether statistically significant differences in this figure of merit are apparent as the number density of microspheres is decreased. An $\alpha$-value of $0.05(P=0.05)$ was used to indicate statistical significance. MATLAB, version R2014B (The MathWorks, Inc.), was used for all computations and statistical analyses.

\section{RESULTS}

\section{Uniform Approach}

The effect of tumor microsphere-number density on $\mathrm{D}_{70}, \mathrm{D}_{90}$, and $\mathrm{D}_{95}$ for the uniform approach is shown in Figure 3A. A clear asymptotic behavior is evident, with maximum variation in $\mathrm{D}_{70}$ in the range of 10,000-70,000 spheres/mL below 5\%. Below 5,000 spheres/mL, $\mathrm{D}_{70}, \mathrm{D}_{90}$, and $\mathrm{D}_{95}$ decrease quickly by almost $20 \%$ at the lowest number density simulated (200 spheres/mL). An interpretation of Figure 3A for different average-tumor absorbed-dose levels is shown in Figure 3B. Figure 3C shows the percentage of simulated clusters occupied by at least 1 microsphere as a function of average number density. At very low number densities $(<1,000$ spheres/mL), insufficient spheres are available and more than $10 \%$ of the anatomic vascular locations capable of forming a cluster will be empty. This effect contributes to the decrease in $\mathrm{D}_{70}$ at a low number density in Figure 3A.

Figure 3D shows dose-volume histograms for 200, 1,000, 10,000 , and 50,000 spheres $/ \mathrm{mL}$. The difference in the dose-volume histogram for 10,000 and 50,000 spheres/mL is minimal. However, a decrease in the slope of the dose-volume histogram between $10,000,1,000$, and $200 \mathrm{spheres} / \mathrm{mL}$ is apparent.

\section{Patient-Based Approach}

Use of the combined macroscopic and microscopic modeling of tumor dosimetry applying the described methods created realistic microscopic absorbed-dose distributions. Figure $4 \mathrm{~A}$ shows a ${ }^{90} \mathrm{Y}$ PET/CT scan of a patient with a left-lobe tumor with peripheral microsphere deposition around a necrotic core (Table 2, tumor 10). Campbell et al. (9) noted the occurrence of peripheral microsphere deposition histologically, which has been reproduced using our combined macroscopic and microscopic modeling technique, as shown in Figure 4B. 


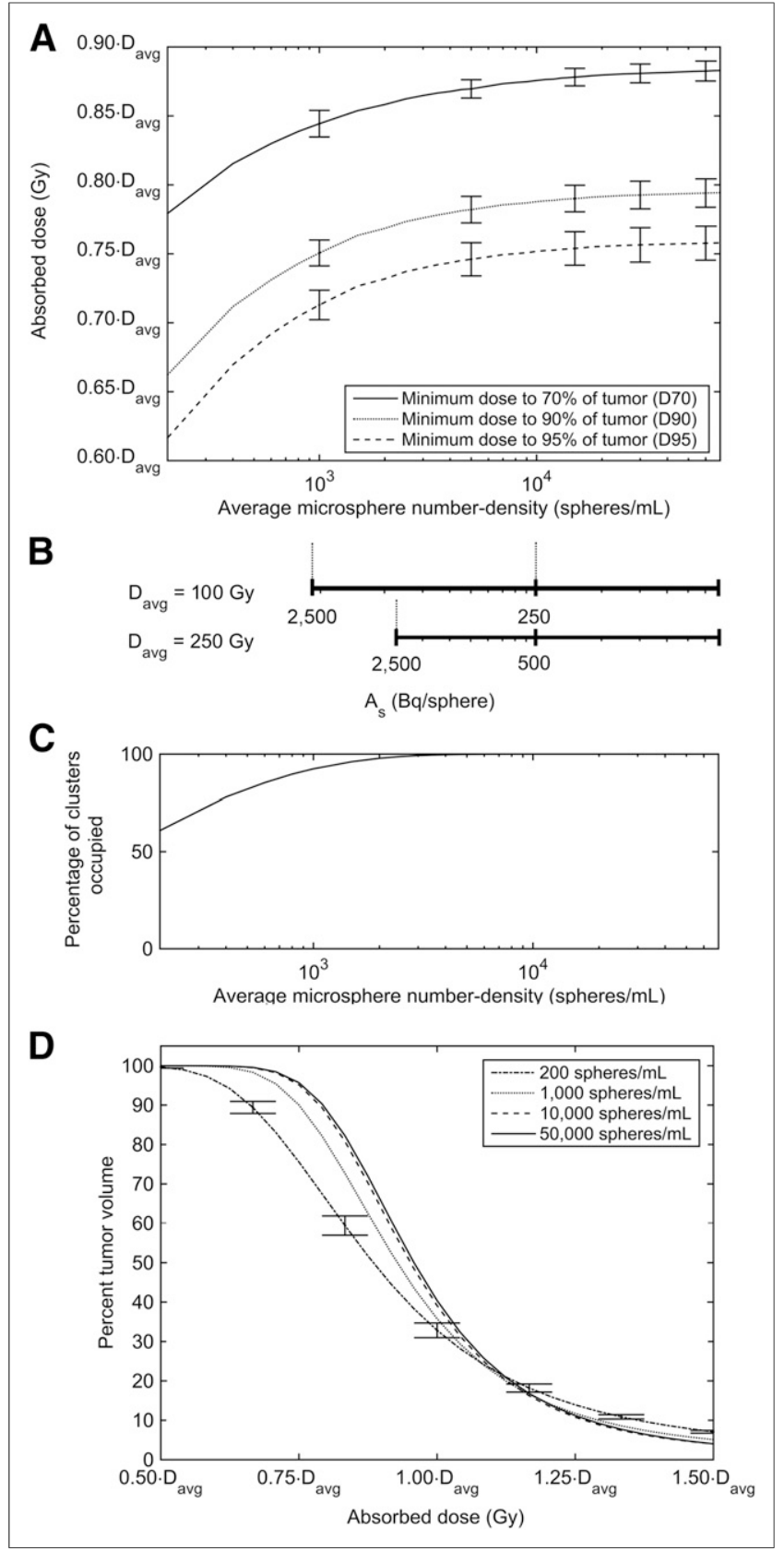

FIGURE 3. Results of simulations using uniform approach in which $D_{\text {avg }}$ is average absorbed dose to tissue volume. (A) $D_{70}, D_{90}$, and $\mathrm{D}_{95}$ as function of average microsphere-number density. Error bars show SD from 20 independent simulations. (B) Specific-microsphereactivity $\left(A_{s}\right)$ horizontal axis labels for $A$ with average absorbed doses of 100 and 250 Gy, as computed using Equation 2. (C) Fraction of microscopic clusters populated by at least 1 microsphere as function of average microsphere-number density (D) Dose-volume histograms for microsphere-number densities of 200, 1,000,10,000, and 50,000 spheres $/ \mathrm{mL}$. For clarity, error bars are shown on 200 spheres $/ \mathrm{mL}$ curve only.

The effect of microsphere-number density on $\mathrm{D}_{70}$ for the patient-based approach using data from ${ }^{90} \mathrm{Y}$ PET/CT is shown in Figure 5. The shape of the $\mathrm{D}_{70}$ curve as a function of number density is consistent despite vastly differing tumor types, sizes,

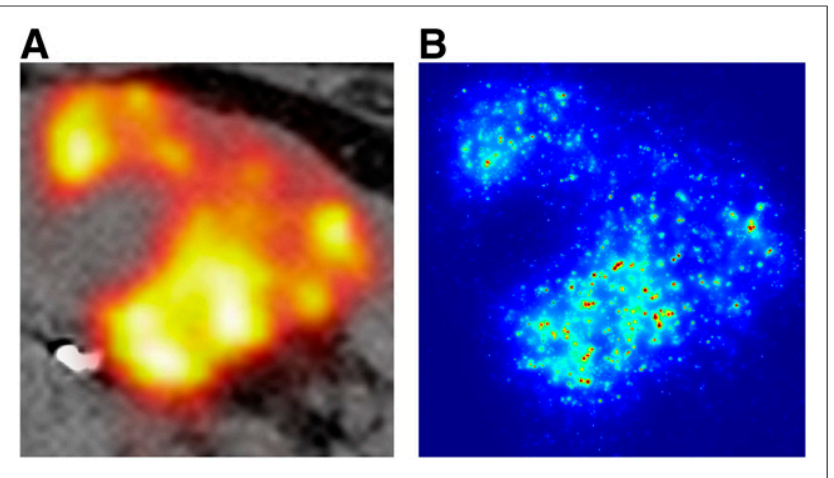

FIGURE 4. (A) ${ }^{90} \mathrm{Y}$ PET/CT of left-lobe tumor reveals peripheral deposition of microspheres. (B) Corresponding microdosimetric absorbed-dose heat map of $100-\mu \mathrm{m}$ slice after population of tumor volume with average number density of 10,000 spheres $/ \mathrm{mL}$.

and macroscopic homogeneities of activity distribution (Table 1). For the tumors simulated, statistically significant differences in $\mathrm{D}_{70}(P<0.05)$ were apparent at less than 20,000 spheres $/ \mathrm{mL}$ when compared with 50,000 spheres/mL $(P<0.01$ for $<18,000$ spheres/mL). $\mathrm{D}_{70}, \mathrm{D}_{90}$, and $\mathrm{D}_{95}$ all decrease as the microspherenumber density decreases, with the greatest obvious changes occurring below 5,000 spheres/mL.

Selected dose-volume histograms are plotted in Figures 6A-6C for tumors 2, 9, and 13, respectively, which represented a range of tumor size, type (Table 1), and macroscopic homogeneity (Fig. 5). In all cases, reductions in the magnitude of the slope of the dosevolume histogram are apparent when the 200 and 1,000 sphere/mL number density are compared with 10,000 spheres/mL. However, these differences are not likely to have clinical significance until the number density decreases below 5,000 spheres/mL as indicated in Figure 5. The effect of macroscopic heterogeneity in activity distribution can be appreciated by comparing Figures $6 \mathrm{~A}-6 \mathrm{C}$ with Figure 3D, which has a much steeper slope because of the uniform macroscopic distribution.

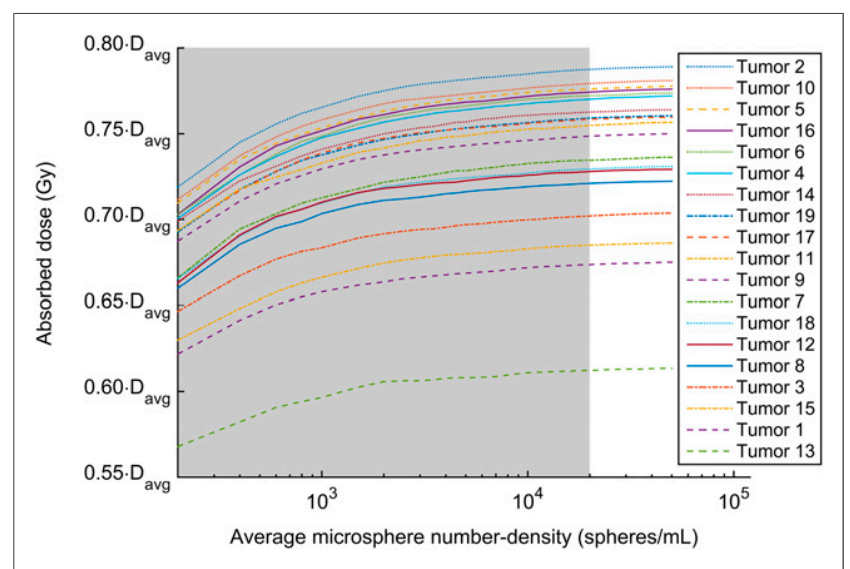

FIGURE 5. $D_{70}$ as function of microsphere-number density for all tumors in Table 2, where $D_{\text {avg }}$ is average absorbed dose to tumor volume. Error bars have been omitted for clarity. Shaded area represents number densities that result in statistically significant differences with $D_{70}$ at 50,000 spheres $/ \mathrm{mL}$. Average absorbed dose, microsphere-number density, and specific activity per sphere are related in Equation 2. 

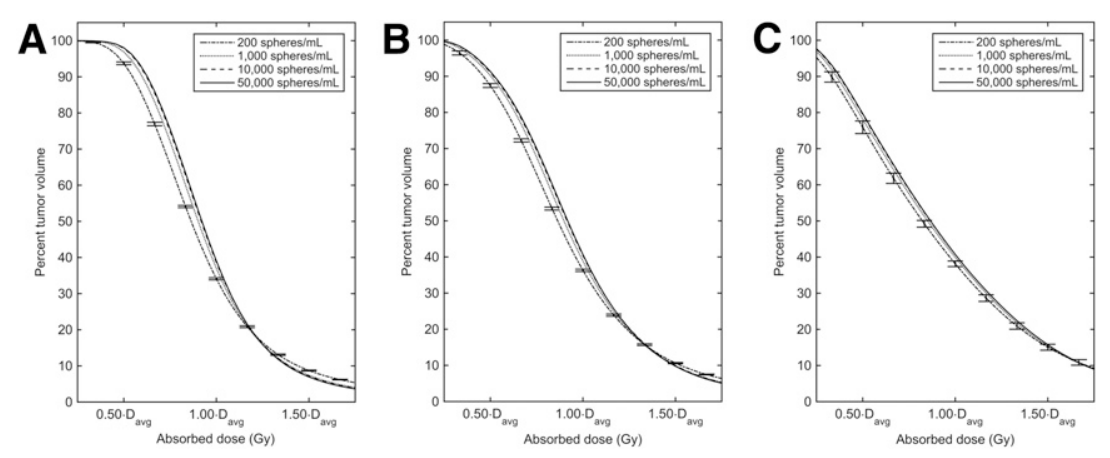

FIGURE 6. Dose-volume histograms for patient-based approach with microsphere-number densities of $200,1,000,10,000$, and 50,000 spheres/mL: tumor $2(A)$, tumor $9(B)$, and tumor 13 (C). Absorbed dose is reported as fraction of average absorbed dose. $\mathrm{D}_{\text {avg }}=$ average absorbed dose to tumor volume.

\section{DISCUSSION}

The presented data show that microdosimetry is affected by variations in tumor microsphere-number density, with higher densities causing a greater percentage of the tumor volume to receive a larger absorbed dose. Although statistically significant differences in $\mathrm{D}_{70}$ were appreciated between 20,000 and 50,000 spheres $/ \mathrm{mL}$, clinical significance is not expected until number density drops below 5,000 spheres $/ \mathrm{mL}$. These results clearly rest on several key assumptions inherent in the model used. The use of ${ }^{90} \mathrm{Y}$ PET/CT to describe macroscopic microsphere distribution is well established (17); however, the validity of extrapolation of Campbell's microscopic characterization of microsphere clusters rests on the assumptions that, first, increasing the number of microspheres does not increase the number of clusters but increases only the number of spheres in each cluster and, second, characterization of size and distance between clusters does not depend strongly on patient-specific microscopic tumor characteristics.

The validity of the first assumption has been noted after histologic analysis of microsphere distribution in patients receiving both glass and resin microspheres (10). Further, this assumption is congruent with an understanding of tumor vascular microanatomy. Microspheres are deposited in capillary beds in a periarteriolar fashion, creating clusters that preferentially form in regions around vascular abnormalities. Since additional microspheres will not alter this process, it is logical that the effect of more microspheres would only be an increased number of spheres per cluster, up to the point at which complete occlusion of the capillary bed occurs. Conversely, if the microsphere-number density is very low, some microvascular areas capable of forming clusters may be left empty, simply because there are not enough spheres to reach them. Our simulation accounted for empty clusters, which influenced the decrease in dose metrics at very low number densities $(<1,000$ spheres/mL) as shown in Figure 3.

The second assumption cannot be fully supported at this time since it is likely that tumor type, tumor size, and prior treatments such as transarterial chemotherapy will create microscopic microsphere cluster distributions different from those reported by Campbell et al. (9). However, the overall behavior of the results should be preserved despite changes in microsphere cluster distribution, with lower microsphere-number densities resulting in higher absorbed-dose inhomogeneity. What may potentially change is the point at which this increased inhomogeneity becomes clinically significant. A patient-specific evaluation of the magnitude of this effect, however, is not feasible.

The presented results about differences in microsphere-number density may partially explain the treatment-planning differences between glass and resin microspheres (Table $1)$. It is in this context that the results in Figures 3, 6, and 7 should be interpreted. For example, consider a patient of average height and weight $(172 \mathrm{~cm}, 70 \mathrm{~kg})$ receiving lobar therapy with a lobar volume of 1,000 $\mathrm{cm}^{3}$ and $25 \%$ tumor infiltration. Using the body-surface-area (4) treatment-planning model for radioembolization with resin microspheres, a 1.25-GBq treatment activity is recommended. Conversely, treatment planning using glass microspheres is based on an 80- to 150 -Gy average dose endpoint (4), or 1.64-3.09 GBq. In this scenario, we will assume that the average tumor-absorbed dose after infusion of $1.25 \mathrm{GBq}$ of resin microspheres is $100 \mathrm{~Gy}$. If tumor and normal-tissue uptake characteristics hold, treatment with glass microspheres will result in a 13- to 250-Gy average tumor-absorbed dose, corresponding to a $1.64-$ to $3.09-\mathrm{GBq}$ treatment activity. Consider in Figure 6 that a 100- to 130-Gy increase in average absorbed dose will overcome differences in $\mathrm{D}_{70}$ resulting from any simulated microsphere-number density, in any tumor. Further, the range of number density at which glass spheres can reasonably be used in this scenario to achieve an absorbed dose of $130 \mathrm{~Gy}$ has a lower limit of about 2,500 spheres/mL_an additional important consideration. This limit in usable range further decreases differences in $\mathrm{D}_{70}$ at the same average absorbed dose between glass and resin devices, making these differences easier to overcome with an increase in treatment dose. Figure 7 shows a simulated absorbeddose line profile providing a visual representation of this example.

The same $\mathrm{D}_{70}$ for a tumor can be achieved in two ways: by using a greater number of microspheres and a lower absorbed dose or by using fewer microspheres of greater specific activity combined with

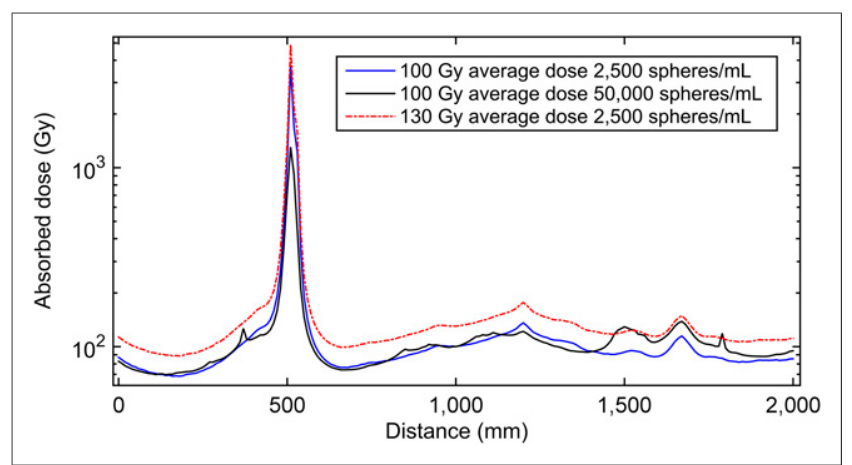

FIGURE 7. Line profile showing absorbed dose across section of tumor with uniform macroscopic deposition. Data for 100-Gy average dose are presented at microsphere-number densities of 50,000 and $2,500 \mathrm{spheres} / \mathrm{mL}$. Characteristic higher peaks and lower troughs at lower microsphere-number densities are apparent. Data are also presented at 2,500 spheres/mL at 130-Gy average absorbed-dose endpoint, consistent with lower limit of recommended treatment activity for radioembolization using glass microspheres. This increase in average tumor-absorbed dose easily overcomes differences due to microsphere-number density. 
an increase in tumor-absorbed dose- that is, by increasing the administered activity. Either approach is expected to provide equivalent tumor control under similar physical and biologic conditions. The findings presented in this paper, therefore, correlate well with clinical efficacy data on glass and resin microspheres and with the relative trade-off between microsphere-number density and treatment dose built into the treatment-planning strategies of these two products.

\section{CONCLUSION}

A lower microsphere-number density may cause a greater fraction of tumor to receive a lower absorbed dose. One can compensate for the effect of number density on clinical dose metrics such as $D_{70}$ by increasing the treatment dose, which is built into the glass treatmentplanning model. Clinicians should be aware of the potential effect of microsphere-number density on absorbed-dose inhomogeneity as it relates to different radioembolization devices. Further, since number density will change with the amount of time that glass microspheres are allowed to decay before use, this factor may also be considered in the treatment-planning process.

\section{DISCLOSURE}

The costs of publication of this article were defrayed in part by the payment of page charges. Therefore, and solely to indicate this fact, this article is hereby marked "advertisement" in accordance with 18 USC section 1734. Alexander S. Pasciak and Yong C. Bradley received an educational grant from SIRTeX Medical USA in partial support of this project. No other potential conflict of interest relevant to this article was reported.

\section{REFERENCES}

1. Lau W-Y, Kennedy AS, Kim YH, et al. Patient selection and activity planning guide for selective internal radiotherapy with yttrium-90 resin microspheres. Int J Radiat Oncol Biol Phys. 2012;82:401-407.

2. Kennedy A, Nag S, Salem R, et al. Recommendations for radioembolization of hepatic malignancies using yttrium-90 microsphere brachytherapy: a consensus panel report from the radioembolization brachytherapy oncology consortium. Int J Radiat Oncol Biol Phys. 2007;68:13-23.

3. Fox RA, Klemp PF, Egan G, Mina LL, Burton MA, Gray BN. Dose distribution following selective internal radiation therapy. Int $J$ Radiat Oncol Biol Phys. 1991;21:463-467.

4. Salem R, Thurston KG. Radioembolization with ${ }^{90}$ yttrium microspheres: a stateof-the-art brachytherapy treatment for primary and secondary liver malignancies. Part 1: Technical and methodologic considerations. J Vasc Interv Radiol. 2006; 17:1251-1278.

5. Walrand S, Hesse M, Chiesa C, Lhommel R, Jamar F. The low hepatic toxicity per gray of ${ }^{90} \mathrm{Y}$ glass microspheres is linked to their transport in the arterial tree favoring a nonuniform trapping as observed in posttherapy PET imaging. $\mathrm{J}$ Nucl Med. 2014;55:135-140.

6. Gulec SA, Sztejnberg ML, Siegel JA, Jevremovic T, Stabin M. Hepatic structural dosimetry in ${ }^{90} \mathrm{Y}$ microsphere treatment: a Monte Carlo modeling approach based on lobular microanatomy. J Nucl Med. 2010;51:301-310.

7. Yorke ED, Jackson A, Fox RA, Wessels BW, Gray BN. Can current models explain the lack of liver complications in Y-90 microsphere therapy? Clin Cancer Res. 1999;5(suppl):3024s-3030s.

8. Kennedy AS, Kleinstreuer C, Basciano CA, Dezarn WA. Computer modeling of yttrium-90-microsphere transport in the hepatic arterial tree to improve clinical outcomes. Int J Radiat Oncol Biol Phys. 2010;76:631-637.

9. Campbell AM, Bailey IH, Burton MA. Analysis of the distribution of intraarterial microspheres in human liver following hepatic yttrium-90 microsphere therapy. Phys Med Biol. 2000;45:1023-1033.

10. Kennedy ASA, Nutting CC, Coldwell DD, Gaiser JJ, Drachenberg CC. Pathologic response and microdosimetry of ${ }^{90} \mathrm{Y}$ microspheres in man: review of four explanted whole livers. Int J Radiat Oncol Biol Phys. 2004;60:1552-1563.

11. Högberg J, Rizell M, Hultborn R, et al. Heterogeneity of microsphere distribution in resected liver and tumour tissue following selective intrahepatic radiotherapy. EJNMMI Res. 2014;4:48-57.

12. Cashwell ED, Everett CJ. A Practical Manual on the Monte Carlo Method for Random Walk Problems. Los Alamos, NM: LosAlamos National Laboratory; 1959:22-24.

13. McKinney GW, Durkee JW, Hendricks JS. MCNPX Overview. Los Alamos, NM: Los Alamos National Laboratory; 2006.

14. Eckerman KF, Westfall RJ, Ryman JC, Cristy M. Availability of nuclear decay data in electronic form, including beta spectra not previously published. Health Phys. 1994;67:338-345.

15. Photon, Electron, Proton and Neutron Interaction Data for Body Tissues. Bethesda, MD: International Commission on Radiation Units and Measurements; 1992:7-42. ICRU report 46.

16. Kao YH, Steinberg JD, Tay Y-S, et al. Post-radioembolization yttrium-90 PET/ $\mathrm{C}$-part 2: dose-response and tumor predictive dosimetry for resin microspheres. EJNMMI Res. 2013;3:57-69.

17. Willowson KP, Tapner M, Bailey DL. A multicentre comparison of quantitative ${ }^{90} \mathrm{Y}$ PET/CT for dosimetric purposes after radioembolization with resin microspheres. Eur J Nucl Med Mol Imaging. 2015;42:1202-1222. 